\title{
Modeling of a multilane-multiple intersection based on queue theory and standard approach techniques
}

\begin{abstract}
The currently available queue model for multilane traffic control is based on priority discipline. This priority discipline based technique is not suitable to be applied for multilanemultiple intersections, since all vehicles in each lane move simultaneously according to its respective signal phase. In view of this scenario, a new general traffic model for multilanemultiple intersections based on queue theory and standard techniques has been developed. The model framework used in this study is $\mathrm{M} / \mathrm{M} / 1$ single server networks with arbitrarilylinked topology structure. A virtual server for each lane in multiple intersections is introduced in order to control the outgoing vehicles in each lane by their own server. In this study, the decomposition algorithm has been applied to deal with the feed-forward flows and finite buffer between intersections in an open queuing network model. This is achieved by decomposing the network into intersections, where each intersection was analyzed independently in order to obtain the overall model. A real case study has been conducted in one of the busiest streets in Kuala Lumpur. The study shows that the inter-arrival and interdeparture traffic flow follow the exponential distribution which also validates the chosen model. Simulation results show good correlation between the proposed models and real case studies.
\end{abstract}

Keyword: Multilane-multiple intersections; Queue theory; Decomposition method; Virtual server 